\title{
Dietary proteins and food-related reward signals
}

\author{
Katri Peuhkuri,2*, Nora Sihvola' and Riitta Korpela' \\ I Institute of Biomedicine, Pharmacology, University of Helsinki, Helsinki, Finland; ${ }^{2}$ Department of Food and \\ Environmental Sciences, Nutrition, University of Helsinki, Helsinki, Finland
}

\section{Abstract}

Proteins play a crucial role in almost all biological processes. Dietary proteins are generally considered as energy yielding nutrients and as a source of amino acids for various purposes. In addition, they may have a role in food-related reward signals. The purpose of this review was to give an overview of the role of dietary proteins in food-related reward and possible mechanisms behind such effects. Dietary proteins may elicit food-related reward by several different postprandial mechanisms, including neural and humoral signals from the gastrointestinal tract to the brain. In order to exert rewarding effects, protein have to be absorbed from the intestine and reach the target cells in sufficient concentrations, or act via receptors ad cell signalling in the gut without absorption. Complex interactions between different possible mechanisms make it very difficult to gain a clear view on the role and intesity of each mechanism. It is concluded that, in principle, dietary proteins may have a role in food-related reward. However, the evidence is based mostly on experiments with animal models and one should be careful in drawing conclusions of clinical relevance.

Keywords: lactalbumin; amino acids; food hedonics; gut hormones; neuropeptides; serotonin; dopamine

Received: 30 December 2010; Revised: I4 April 2011; Accepted: 10 May 201 I Published: I June 20II

$\mathrm{F}$ ood intake relies on our brain to obtain sensory information about food, to evaluate for desirability, and to choose the appropriate behaviour. What we eat and drink is largely guided by the orosensory and viscerosensory properties of food - i.e. a combination of appearance, taste and smell. Thus, subjective aspects of food hedonics relate to sensory properties (e.g. palatability, smell, texture), cognitive processes (e.g. preferences, aversions, experience) and hedonic perception (pleasure, taste) as reviewed by Sørensen et al. (1). Together, these processes result in personal judgements on whether we like the food or not.

Pleasure is described as a state or feeling of happiness and satisfaction resulting from an experience that one enjoys [for review, see e.g. (2, 3)]. It is a complex neurobiological phenomenon, relying on reward circuitry or limbic activity near the centre of the brain. The biological mechanisms caused by positive emotions such as 'pleasure' or 'enjoyment' are called 'reward' (3). This can be divided neurologically and psychologically into 'liking' (pleasure) and 'wanting' (motivation) and in some cases into 'learning,' too $(2,3)$. Liking and wanting have separable neural substrates, dopamine and opiate system, respectively, which can be manipulated and measured.

The physiology of reward is a complex system originating from reward and motivation circuitries in the central nervous system (CNS) (3). Integral components of the CNS that are involved in reward processes are found in the limbic system and in nerve cells in the ventral tegmental area (VTA) located near the base of the brain. These nerve cells send projections to target regions in the frontal brain section, especially to nucleus accumbens situated deep beneath the frontal cortex. Doing things we enjoy, for example having a tasty meal, boosts the activity of our pleasure and reward system. The essential mediators in these processes include dopamine, serotonin, stress hormones, gamma-aminobutyric acid (GABA) and glutamate, and even endogenous morphine/opioid peptides may be of importance (2).

Postprandial gastrointestinal effects of the diet can indirectly influence these neurobiological processes, independently of the neurochemical content or palatability of the food. The gut-brain axis transmits nutrient information via neural and humoral signals from the 
gastrointestinal tract to the brain. These neurobiological processes include several possible concomitant mechanisms, depending on what and how much we eat. What we know about these mechanisms is based mostly on studies with glucose and sucrose.

Humans have an innate preference for sweet taste and excessive appetite for sugar and fat containing products may cause overeating thus inflicting on health problems. The challenge is to find new concepts for palatable healthy foods with similar sugar-like rewarding properties. Among other possibilities, attention has focused on dietary proteins. The influence of dietary proteins on the hedonic system, however, is poorly understood. Orosensory properties of a protein-containing diet seem to be of minimal importance to food preferences, but the postprandial influence of dietary proteins on the food reward system is an interesting issue. Proteins consist of amino acids joined together by peptide bonds. Dietary proteins are degraded back to peptides and amino acids by digestive enzymes during the transit through the small intestine. Even if proteins can be absorbed as such, they are mostly absorbed and utilised as amino acids and diand tripeptides (4). Single amino acids, as well as di- and tripeptides, are easily and efficiently transported into the intestinal cells from the lumen by specific transporters and subsequently released into the blood for absorption by other tissues. Released amino acids are used mostly for protein synthesis, but some of them are converted to glucose through gluconeogenesis or fed into the citric acid cycle. These peptides and amino acids as such, as well as the triggered metabolic cascade, may influence the hedonic response to food.

This paper reviews the evidence on the postprandial effect of ingested, digested and absorbed dietary proteins on the food reward system. Physiological mechanisms that control appetite and food intake are excluded from this review.

\section{The role of the taste of proteins}

Although humans react to the taste and texture of the food rather than its chemical content, carbohydrates are clearly preferred over proteins and especially over amino acids. It is not a question of the presence or absence of sweet taste, since mice prefer a glucose solution over an amino acid solution even when sweet-taste amino acids, such as L-glutamine, L-alanine and L-threonine (5), were employed or when sweet-blind knockout mice were used (6). L-glutamate is a multifunctional amino acid in most organs and tissues and as monosodium L-gluatame it is one of the main components of umami taste [for review see Kondoh and Torii (7)]. In a recent experiment, Uematsu et al. (8), rats preferred sucrose over monosodium L-glutamate solution. They also noticed that neural pathways that process tastes of sweet (sugar) and umami (amino acid glutamate) are similar and may even have interaction, although the following activation of neural networks may differ in the higher brain regions resulting in behavioural differences as observed in tests with rats measuring 'wanting.'

Sweet-tasting proteins, such as brazzein, thaumatin, monelin, curculin, mabinlin, miraculin and pentadin, have been reviewed by Kant (9) and Temussi (10). All of these proteins have been isolated from tropical plants. Humans detect the sweet taste of these proteins with the same taste receptor cells clustered in taste buds as the sweet taste of sucrose. However, the mechanism of interaction and taste characteristics of these sweet proteins may differ from that of conventional sweeteners (11). The potential applications of these proteins are lowcalorie, sweet-tasting so called light products and weight management.

Studies comparing the pleasantness of dietary proteins are scanty. Casein is one of the major protein fractions in milk and intact casein is favoured over casein hydrolysates. A study of Field et al. (12) showed that hydrolysed casein is avoided dose-dependently by mice, mimicking the corresponding aversion reactions of many people (13). If the casein hydrolysate concentration exceeds $30 \%(\mathrm{w} / \mathrm{w})$ in the diet, consumption is reduced in most cases compared to a diet containing intact casein. This can be explained by the bitter taste of some peptides and amino acids that are released during hydrolysis. The taste of amino acids varies and depends at least partly on their structural configuration (14). Sweet amino acids are primarily found among the D-series of amino acids, whereas bitter tasting amino acids are generally within the L-series. L-tryptophane and L-tyrosine are the bitterest amino acids, whereas D-tryptophane is the sweetest. By contrast, except for the sweet-tasting esters of aspartic acid, peptides are neutral or bitter in taste with no relationship to their configuration. The taste intensity of peptides varies depending on the type of protein and enzyme used and does not appear to be dependent on the amino acid sequence as reviewed by Maehashi and Huang (15).

Pérez et al. (16) found no differences in the preference pattern between the intragastric infusion of $10 \%$ carbohydrate and $10 \%$ protein (calcium caseinate) in a twobottle choice test with rats. However, when the oral and gastric preloads of either protein or carbohydrate were combined, the preference patterns were significantly modified, which was hardly seen at all after an oral preload only. This indicates that both orosensory and viscerosensory signals are generated and their combined action may be cumulative. Thus, if taste is not considered, proteins and carbohydrate may have parallel preference patterns.

To summarise, orosensory properties of proteins and especially those of amino acids are generally not considered very pleasant neither by animals nor by humans. 
Even so, proteins, peptides and even bitter-tasting amino acids may have postprandial effects that influence the total hedonic value of protein.

\section{The site of protein action - visceral, neural and humoral mechanisms}

The most important sites of protein action for food hedonics are the metabolic signal chains triggered by ingestion processes in the gut. These postprandial metabolic processes are affected by the quality, quantity and structure of dietary proteins. For example, although casein and casein hydrolysates have nearly equivalent nutritive value, their postprandial metabolic differences modify the gastrointestinal activities, and thus possibly affect the development of food preferences (12). There seems to be no differences in the gastric emptying rate between casein and casein hydrolysates in humans, but the absorption of amino acids from hydrolysed casein is faster, which produced more gastric secretion and induced a greater amount of glucose-dependent insulinotropic polypeptide (17). The gastrointestinal transit time is shorter for a diet containing casein than a diet containing hydrolysed casein, which may be, at least partly, due to the opioid activity of peptides released during in vivo digestion of casein (18). The role of gastric motility in food hedonics, however, remains to be clarified.

Basically, there are two possible routes of action for protein to modulate food-related reward signals: either by sending neural signals via the vagus nerve or by triggering the release of gastrointestinal hormones, such as cholecystokinin, ghrelin and insulin. Irrespective of the mechanism in question, these postprandial effects have been documented to modulate subsequent food preferences. The influence of these intestinal metabolic processes on food preferences can be strong even with the sweet-tasting sucrose. Ren et al. (6) showed that sweetblind knockout mice (trpm $5^{-1-}$ ) develop a preference for D-glucose compared with isocaloric L-serine independently of the perception of sweetness. They also found a close relationship between glucose oxidation and tasteindependent nutrient intake levels. These higher intake levels were more markedly associated with glucose oxidation rates than with increases in blood glucose. According to the authors, this establishes the influence of metabolism.

The ascending neural pathway from the gut is the afferent vagus nerve. It projects to the nucleus of the solitary tract, which conveys visceral information to the brain. Apart from being influenced by physical visceral stimuli, such as gastric expansion, the vagus nerve is also activated by chemical stimulation. Electrophysiological studies have shown that the administration of L-glutamate as monosodium glutamate into the stomach or the small intestine activates branches of the vagus nerve, whereas the administration of glucose has a minor effect (19). L-glutamate is a multifunctional amino acid, which is the most prevalent amino acid in almost all dietary proteins and can also be considered as one of the key molecules in cell metabolism. Ingestion of glutamate has minimal influence on the concentration of glutamate in the blood, suggesting that blood glutamate levels do not provide essential information about the ingested glutamate. Stimulation of recently identified L-glutamate receptors by luminal L-glutamate activates vagal afferent nerve fibres via the local production and release of nitric oxide and the release of serotonin, which supports intracellular communication between mucosal cells and the vagus nerve with nitric oxide and serotonin as messengers (20). Functional magnetic resonance imaging (fMRI) in rats has further revealed that several forebrain regions are activated after ingestion of L-glutamate (21). L-glutamate-induced forebrain activation was strongly suppressed by vagatomy, which convinces the importance of the neural route. Glucose, instead, has stronger humoral signal routes, and vagatomy has only a minor influence on the forebrain activation after glucose ingestion (19). The ability of the gastric afferent to respond to any other amino acids, however, seems low compared to glutamate (22).

The chemical composition of ingested food influences the release of gut hormones from intestinal enteroendocrine cells. The association of these peptide hormones, such as cholecystokinin, ghrelin and insulin from the pancreas, with the control of food intake and satiety is strong (23). However, these hormones exert multiple physiological effects that not only influence food intake and the digestion of nutrients but also emotion and food preferences (24). Several gut hormones can cross the blood-brain barrier and enter the brain. For example, insulin can interact with some signal-transduction receptors in the hippocampus region [for review, see GómezPinilla (25)].

Dietary proteins have a clear influence on the release of gut hormones, such as cholecystokinin, glucagon-like peptide 1, peptide YY, pancreatic polypeptide, insulin, and possibly also on the release of ghrelin and glucosedependent polypeptide (23). However, the exact role and mechanism of action of these peptide hormones in food hedonics is not clear. Plasma insulin is considered to be important, especially for mediating the effects of the postprandial glucose load (19). Besides dietary amino acids, proteins also stimulate the secretion of insulin and glucagon, and the influence of different proteins and amino acids to this secretory action vary $(26,27)$.

In addition to gut hormones, other hormones or hormone-like compounds also may modulate food hedonics. A study of Nakamura et al. showed that besides the postingestive rise of blood glucose and insulin levels, plasma leptin levels may modulate the sweet taste 
sensitivity as well (28). The recognition thresholds for sweet taste exhibit a diurnal variation similar to the variation of plasma leptin levels, which suggests a mechanistic connection between these two variables in humans. The lowest thresholds were noticed in the morning and the highest thresholds at night. This diurnal variation was not observed in thresholds for any other taste stimuli, such as sodium chloride, citric acid, quinine and mono-sodium glutamate. Unfortunately, this interesting study did not test any other dietary proteins, peptides, or amino acids.

To summarise, there are routes for gastrointestinal neural and humoral signals to encode the food-related reward system that are independent of taste signals or caloric load. Proteins share these routes with other nutrients, but the intensity may differ. Information on dietary proteins is converted to the brain via both the vagus nerve and gut hormones, especially insulin. Evidence is mostly from animal models and clinical relevance needs to be established later.

\section{The protein components as precursors of brain reward mediators}

\section{The serotonin pathway}

Brain serotonin is involved in a broad range of different physiological and behavioural functions. There is clear evidence that the reward-related areas in the brain and rewarding experiences are linked with the serotonergic system, as recently reviewed by Kranz et al. (29). The authors concluded from numerous reviewed studies in rodents and humans that even if the reward behaviour can be modified due to altered serotonergic action induced by an increase or decrease in serotonin function, the clinical impact of manipulating extracellular serotonin levels must be interpreted with caution due to the various feedback mechanisms that result in a readjustment of the transmitter systems.

The rate of serotonin (5-hydroxytryptamine, 5-HT) synthesis in the brain depends on local concentrations of its amino acid precursor, L-tryptophan. Brain tryptophan concentrations, in turn, reflect uptake from circulation, which occurs via a blood-brain barrier transport carrier shared among several large, neutral amino acids (LNAA) including tryptophan, phenylalanine, tyrosine, leucine, isoleucine and valine. Transport is saturable and competitive. Hence, tryptophan uptake is modified by altering the blood concentrations of either tryptophan or any other of its LNAA competitors.

Certain foods or changes in the composition of dietary amino acids may modify brain function by interfering with neurotransmitter synthesis. Tryptophan is an essential amino acid that must be obtained from dietary proteins. Choi et al. (30) showed the tryptophan concentration and serotonin synthesis in rat brain circuits being remarkably sensitive to the presence of a particular protein in a meal. A large rise in cortex tryptophan occurred after $\alpha$-lactalbumin consumption, followed by a corresponding increase in serotonin synthesis. The $\alpha$ lactalbumin is a whey protein with a very high tryptophan concentration (31). The influence of dietary soy protein or carbohydrate (no protein) was significantly smaller on tryptophan concentration, and after ingestion of other types of protein, such as zein, casein or gluten, tryptophan concentration dropped to very low levels (relative to the fasting state) in the brain circuits. The potential of $\alpha$ lactalbumin to induce serotonin synthesis in the brain has been confirmed in an extensive series of clinical studies by Markus (32).

Dietary carbohydrates also influence the blood concentration of tryptophan by lowering the concentrations of the competitors of tryptophan without affecting tryptophan itself (33). Dietary carbohydrates induce an elevation of glucose and insulin, which, with the exception of tryptophan, causes the LNAAs to be taken up into the skeletal muscles for conversion into proteins. The increase of insulin causes free fatty acids to be stripped away from circulating albumin in the blood, thus promoting the take up of free fatty acids by adipocytes. Unbound albumin binds loosely to tryptophan, which is thus prevented from being taken up in the periphery and is available in the brain. The influence of carbohydrates on the increase in the ratio of tryptophan to other LNAAs is $20-45 \%$, whereas the influence of dietary $\alpha$ lactalbumin is $50-70 \%$. Pure tryptophan increases this ratio to over $100 \%$ (32).

The importance of brain serotonin in the regulation of stress, mood and eating behaviour has been demonstrated by several clinical interventions, as reviewed by Markus (32). Increased serotonin elevates mood and reduces the desire to eat, especially the desire to eat sweet, carbohydrate-rich foods (34). However, there are individual differences in the sensitivity and strength of the influence of $\alpha$-lactalbumin on the serotonin effects in the brain. Even if a significant increase in available tryptophan and clear signs of induction of serotonin synthesis are noticed, the clinical outcome may be minimal. The effects seem to be rather modest, especially in healthy subjects, but appear to be more probable in vulnerable subjects or under stressful conditions.

The number of studies on modifying the hedonic responses to food by dietary tryptophan is small. A single oral dose of a tryptophan-rich modified food hedonics and reduced the preference for sweet foods in young adults with high trait anxiety (35). In this study, tryptophan was given as a single dose study meal containing $20 \mathrm{~g}$ of $\alpha$-lactalbumin, of which $2.6 \%$ was tryptophan. Previously, Beulens et al. (36), however, found dietary $\alpha$-lactalbumin to have no effect either on macronutrient preference or on food intake. In this 
study $\alpha$-lactalbumin supplement was combined with a regular diet containing $15 \%$ of energy from proteins. In such a diet, tryptophan has too many LNAA competitors to struggle the transport across the blood-brain barrier.

No evidence of the long-term effects of dietary modification of blood tryptophan concentrations on mood, appetite and food hedonics is available, as far as we know. In a study in rats, Orosco et al. (37) found that if a tryptophan-rich diet was continued for 3 to 6 weeks, the serotonin release in the medial hypothalamus was reduced and the sucrose consumption doubled while no change occurred in the control animals receiving caseinbased diet. In the same study, the acute effect of an $\alpha$ lactalbumin rich diet was the opposite - the sucrose consumption decreased. The authors suggested that chronic activation of the serotonin system by long-term $\alpha$-lactalbumin diet may trigger rewarding effects and excess tryptophan may be used in protein metabolism or converted into secondary metabolites of the kynurenine pathway instead of stimulating serotonin synthesis.

To sum up, changes in the brain serotonin are mostly due to the availability of its precursor, tryptophan, entering the brain, which can be influenced by diet as established in several nice clinical trials. The serotonin concentration is clearly associated with rewarding behaviour, sensitivity to mood disorders, stress and appetite. Although there are indications that the serotonergic system impacts food hedonics and is thus able to modify the liking and preferences for food, the clinical relevance of this route to food preferences, either acute or longterm, remains to be clarified.

\section{The dopamine pathway}

The role of the brain dopamine system in mediating food reward is well established in several studies using sucrose and glucose. Tasting palatable foods elevates dopamine levels in the brain region known as nucleus accumbens. Blocking dopamine receptors with suitable antagonists diminishes the hedonic value of sweet-tasting nutrients such as sucrose (38). The brain dopamine reward system can be activated even in the absence of taste transduction. The development of the preference for sucrose is independent of the activation of taste receptors, as shown with sweet-blind knockout mice (trpm5 $5^{-1-}$ mice) (39). Dietary sucrose, except for the non-caloric sweetener, sucralose, induced dopamine release in the rewardprocessing regions of mice brain, which indicates that caloric intake can produce measurable increases in dopamine levels in the brain reward circuits. Stimulation of dopamine release by intragastric glucose seemed to depend on glucose utilisation, which was seen by lower dopamine levels after administration of an anti-metabolic glucose analogue (6). The role of endocannabinoid system in the peripheral control of metabolism, at least that of lipids and glucose, as well as in energy expenditure has been reviewed previously (40).

Catecholamines (i.e. dopamine, norepinephrine and epinephrine) are synthesised from amino acid tyrosine. The rates of synthesis and the release of these neurotransmitters are directly modified by the brain concentrations of their precursors, tyrosine and phenylalanine (41). Tyrosine is the preferred substrate and ingested phenylalanine can be rapidly hydroxylated to tyrosine in the liver and provided to the circulation. Brain tyrosine uptake depends on the serum levels of tyrosine and its LNAA competitors that, in turn, are influenced by diet. The brain levels of threonine, histidine, or glutamine can control the rates at which neurons synthesise other neurotransmitters such as glycine, histamine, or GABA.

Little is known about the effects of different dietary proteins on dopamine levels. Different sources of protein in a single meal caused changes in cortical tyrosine concentrations in rats but at a much smaller scale than was seen in tryptophan concentration (30). However, even if tyrosine levels in the brain paralleled the changes in the serum tyrosine/LNAA ratio fairly well, and the concentration of tyrosine doubled in the brain after a casein-containing meal, no changes were noticed in the dopamine synthesis rate in brain circuits. Previously, in a chronic dietary paradigm, in which the dietary protein content was varied, twofold differences in brain tyrosine concentrations were associated with significant changes in hypothalamic dopamine synthesis (41).

To summarise, the synthesis of catecholamines, such as dopamine, is dependent of its amino acid precursors. The role of dopaminergic signalling in reward processes is clear. However, the influence of dietary proteins on catecolamine neurons seems only modest, at the most.

\section{Opioid peptides}

Peptides binding to opioid receptors in the brain are known as opioid peptides. Drugs mimicking the effects of these peptides are opiates and opioids. Opioid peptides can be formed in the gut as the result of in vivo hydrolysis of dietary proteins, but they may also be absorbed from partially digested food (42). Some milk peptides, for example, have an affinity for an opiate receptor and opiate-like effects. Several types of opioid-agonists as well as antagonist peptides have been characterised, but the major opioid peptides are fragments of $\beta$-caseins. Once absorbed into blood, some of these peptides can cross the blood-brain barrier, travel to the brain and various other organs, and elicit pharmacological properties similar to opium or morphine.

The physiological effects of these peptides vary. Brain opioid peptide systems have an important role in motivation, emotion, the response to stress and pain and the control of food intake. Individual receptors are responsible for these specific physiological effects; i.e. the 
$\mu$-receptor for emotional behaviour and suppression of intestinal motility and the $\kappa$-receptor for sedation and food intake [for review, see Ganapathy and Miyauchi (42)].

Opioid peptides are involved in the food reward system and have a role especially in the palatability of preferred foods [for review Barbano and Cador (43)]. Endogenous opioids are released as food is eaten, and this is thought to enhance the pleasure of eating. The opioid system can be modified by the consumption of highly palatable, sugar- and fat-containing foods, but the influence of dietary proteins on the opioid systems is not known. In general, however, opioid peptides have limited physiological activity.

\section{Physiological significance - conclusions}

With respect to food-related pleasure, sensations such as taste, smell and texture are very important and their potency should not be understated. Nevertheless, food preferences are clearly documented to be much more intricate than the orosensory properties of food. Dietary proteins may elict food-related reward processes by several different postprandial mechanisms. These can basically be divided into three categories: neural signals via the vagus nerve, metabolic signals mediated by gastrointestinal hormones, and possible other metabolites and modification of neurotransmitters in the brain by providing suitable amino acid precursors. The intensity of each of these routes seems to be modest. However, the complexity of the interactions between the mechanisms makes it very difficult to gain a clear view of their respective roles in reward processes. Studies investigating those complexities are scarce. Furthermore, the evidence is based mostly on experiments with animal models and one should be careful in drawing conclusions of clinical relevance.

These protein-mediated mechanisms do not mediate acute immediate reactions. Their influence is slower since the digestion and absorption of nutrients requires time. Thus, their importance is more evident in the signs of postprandial well-being. In general, pleasure can reduce stress and the postprandial food reward can thus be partly due to its stress-relieving capacity. Pleasure may involve substances that possess calming and anxiolytic properties, thereby facilitating feelings of well-being and relaxation, which can also be detected on the neurochemical level. Future studies could focus on unifying the hypotheses of stress-relieving processes in relation to food-related reward responses to gain a detailed understanding of the physiology of dietary proteins.

\section{Conflicts of interest and funding}

The authors declare no conflict of interest regarding this review. The authors have not received any funding or benefits from industry but N.S. gets funding from nonprofit SalWe Ltd research programme Mind and Body.

\section{References}

1. Sørensen LB, Møller P, Flint A, Martens M, Raben A. Effect of sensory perception of foods on appetite and food intake: a review of studies on humans. Int $\mathbf{J}$ Obes Relat Metab Disord 2003; 27: 1152-66.

2. Berridge KC. Food reward: brain substrates of wanting and liking. Neurosci Biobehav Rev 1996; 20: 1-25.

3. Esch T, Stefano GB. The neurobiology of pleasure, reward processes, addiction and their health implications. Neuro Endocrinol Lett 2004; 25: 235-51.

4. Hara H, Funabiki R, Iwata M, Yamazaki K. Portal absorption of small peptides in rats under unrestrained conditions. J Nutr 1984; 114: 1122-9.

5. Bachmanov AA, Beauchamp GK. Amino acid and carbohydrate preferences in C57BL/6ByJ and 129P3/J mice. Physiol Behav 2008; 93: 37-43.

6. Ren X, Ferreira JG, Zhou L, Shammah-Lagnado SJ, Yeckel $\mathrm{CW}$, de Araujo IE. Nutrient selection in the absence of taste receptor signaling. J Neurosci 2010; 30: 8012-23.

7. Kondoh T, Torii K. Brain activation by umami substances via gustatory and visceral signalling pathways, and physiological significance. Biol Pharm Bull 2008; 31: 1827-32.

8. Uematsu A, Tsurugizawa T, Kitamura A, Ichkawa R, Iwatsuki $\mathrm{K}$, Uneyama H, et al. Evaluation of the 'liking' and 'wanting' properties of umami compounds in rats. Physiol Behav 2011; 102: $553-8$

9. Kant R. Sweet proteins - potential replacement for artificial low calorie sweeteners. Nutr J 2005; 4: 5.

10. Temussi PA. Natural sweet macromolecules: how sweet proteins work. Cell Mol Life Sci 2006; 63: 1876-88.

11. Dotson CD, Spector AC. Behavioral discrimination between sucrose and other natural sweeteners in mice: implications for the neural coding of T1R ligands. J Neurosci 2007; 27: 11242 53.

12. Field KL, Kimball BA, Mennella JA, Beauchamp GK, Bachmanov AA. Avoidance of hydrolyzed casein by mice. Physiol Behav 2008; 93: 189-99.

13. Pedrosa M, Pascual CY, Larco JI, Esteban MM. Palatability of hydrolysates and other substitution formulas for cow's milkallergic children: a comparative study of taste, smell, and texture evaluated by healthy volunteers. J Investig Allergol Clin Immunol 2006; 16: 351-6.

14. Temussi PA. Sweet, bitter and umami receptors: a complex relationship. Trends Biochem Sci 2009; 34: 296-302.

15. Maehashi K, Huang L. Bitter peptides and bitter taste receptors. Cell Mol Life Sci 2009; 66: 1661-71.

16. Pérez C, Ackroff K, Sclafani A. Carbohydrate- and proteinconditioned flavor preferences: effects of nutrient preloads. Physiol Behav 1996; 59: 467-74.

17. Calbet JA, Holst JJ. Gastric emptying, gastric secretion and enterogastrone response after administration of milk proteins or their peptide hydrolysates in humans. Eur J Nutr 2004; 43: 12739.

18. Mihatsch WA, Franz AR, Kuhnt B, Högel J, Pohlandt F. Hydrolysis of casein accelerates gastrointestinal transit via reduction of opioid receptor agonists released from casein in rats. Biol Neonate 2005; 87: 160-3.

19. Tsurugizawa T, Uematsu A, Nakamura E, Hasumura M, Hirota $\mathrm{M}$, Kondoh $\mathrm{T}$, et al. Mechanisms of neural response to gastrointestinal nutritive stimuli: the gut-brain axis. Gastroenterology 2009; 137: 262-73. 
20. Kondoh T, Mallick HN, Torii K. Activation of the gut-brain axis by dietary glutamate and physiologic significance in energy homeostasis. Am J Clin Nutr 2009; 90: S832-7.

21. Uematsu A, Tsurugizawa T, Uneyama H, Torii K. Brain-gut communication via vagus nerve modulates conditioned flavor preference. Eur J Neurosci 2010; 31: 1136-43.

22. Uneyama H, Niijima A, San Gabriel A, Torii K. Luminal amino acid sensing in the rat gastric mucosa. Am J Physiol Gastrointest Liver Physiol 2006; 291: G1163-70.

23. Karhunen LJ, Juvonen KR, Huotari A, Purhonen AK, Herzig KH. Effect of protein, fat, carbohydrate and fibre on gastrointestinal peptide release in humans. Regul Pept 2008; 149: 70-8.

24. Sternini C, Anselmi L, Rozengurt E. Enteroendocrine cells: a site of 'taste' in gastrointestinal chemosensing. Curr Opin Endocrinol Diabetes Obes 2008; 15: 73-8.

25. Gómez-Pinilla F. Brain foods: the effects of nutrients on brain function. Nat Rev Neurosci 2008; 9: 568-78.

26. Ji H, Bachmanov AA. Differences in postingestive metabolism of glutamate and glycine between C57BL/6ByJ and 129P3/J mice. Physiol Genomics 2007; 31: 475-82.

27. Young A. Inhibition of glucagon secretion. Adv Pharmacol 2005; 52: 151-71.

28. Nakamura Y, Sanematsu K, Ohta R, Shirosaki S, Koyano K, Nonaka K, et al. Diurnal variation of human sweet taste recognition thresholds is correlated with plasma leptin levels. Diabetes 2008; 57: 2661-5.

29. Kranz GS, Kasper S, Lanzenberger R. Reward and the serotonergic system. Neuroscience 2010; 166: 1023-35.

30. Choi S, Disilvio B, Fernstrom MH, Fernstrom JD. Meal ingestion, amino acids and brain neurotransmitters: effects of dietary protein source on serotonin and catecholamine synthesis rates. Physiol Behav 2009; 98: 156-62.

31. Heine W, Radke M, Wutzke KD, Peters E, Kundt G. Alphalactalbumin-enriched low-protein infant formulas: a comparison to breast milk feeding. Acta Paediatr 1996; 85: 1024-8.

32. Markus CR. Dietary amino acids and brain serotonin function; implications for stress-related affective changes. Neuromolecular Med 2008; 10: 247-58.

33. Wurtman RJ, Fernstrom JD. Control of brain monoamine synthesis by diet and plasma amino acids. Am J Clin Nutr 1975; 28: 638-47.
34. Wurtman J, Wurtman R, Mark S, Tsay R, Gilbert W, Growdon J. d-Fenfluramine selectively suppresses carbohydrate snacking by obese subjects. Int J Eat Disord 1985; 4: 89-99.

35. Verschoor E, Finlayson G, Blundell J, Markus CR, King NA. Effects of an acute alpha-lactalbumin manipulation on mood and food hedonics in high- and low-trait anxiety individuals. $\mathrm{Br}$ J Nutr 2010; 104: 595-602.

36. Beulens JW, Bindels JG, de Graaf C, Alles MS, WoutersWesseling W. Alpha-lactalbumin combined with a regular diet increases plasma Trp-LNAA ratio. Physiol Behav 2004; 81: 585 93.

37. Orosco M, Rouch C, Beslot F, Feurte S, Regnault A, Dauge V. Alpha-lactalbumin-enriched diets enhance serotonin release and induce anxiolytic and rewarding effects in the rat. Behav Brain Res 2004; 148: 1-10.

38. Wise RA. Forebrain substrates of reward and motivation. J Comp Neurol 2005; 493: 115-21.

39. de Araujo IE, Oliveira-Maia AJ, Sotnikova TD, Gainetdinov RR, Caron MG, Nicolelis MA, et al. Food reward in the absence of taste receptor signaling. Neuron 2008; 57: 930-41.

40. Di Marzo V, Ligresti A, Cristino L. The endocannabinoid system as a link between homeostatic and hedonic pathways involved in energy balance regulation. Int J Obes 2009; 33: S1824.

41. Fernstrom JD, Fernstrom MH. Tyrosine, phenylalanine, and catecholamine synthesis and function in the brain. J Nutr 2007; 137: S1539-47.

42. Ganapathy V, Miyauchi S. Transport systems for opioid peptides in mammalian tissues. AAPS J 2005; 7: E852-6.

43. Barbano MF, Cador M. Opioids for hedonic experience and dopamine to get ready for it. Psychopharmacology (Berl) 2007; 191: 497-506.

\footnotetext{
*Katri Peuhkuri, PhD

Institute of Biomedicine

Pharmacology

P.O. Box 63, FIN-000 I4, University of Helsinki

Helsinki, Finland

Tel: +3589 19| 25331

Fax: +3589 |9| 25364

Email: katri.peuhkuri@helsinki.fi
} 